\title{
Different Characterizations of Large Submodules of QTAG-Modules
}

\author{
Fahad Sikander, ${ }^{1}$ Alveera Mehdi, ${ }^{2}$ and Sabah A. R. K. Naji ${ }^{3}$ \\ ${ }^{1}$ College of Science and Theoretical Studies, Saudi Electronic University, Jeddah Branch, Jeddah 23442, Saudi Arabia \\ ${ }^{2}$ Department of Mathematics, Aligarh Muslim University, Aligarh 202002, India \\ ${ }^{3}$ Department of Mathematics, Al Bayda University, Al Bayda, Yemen
}

Correspondence should be addressed to Alveera Mehdi; amehdi.wc@amu.ac.in

Received 24 July 2016; Accepted 5 December 2016; Published 3 January 2017

Academic Editor: Shaofang Hong

Copyright (C) 2017 Fahad Sikander et al. This is an open access article distributed under the Creative Commons Attribution License, which permits unrestricted use, distribution, and reproduction in any medium, provided the original work is properly cited.

\begin{abstract}
A module $M$ over an associative ring $R$ with unity is a QTAG-module if every finitely generated submodule of any homomorphic image of $M$ is a direct sum of uniserial modules. The study of large submodules and its fascinating properties makes the theory of QTAG-modules more interesting. A fully invariant submodule $L$ of $M$ is large in $M$ if $L+B=M$, for every basic submodule $B$ of $M$. The impetus of these efforts lies in the fact that the rings are almost restriction-free. This motivates us to find the necessary and sufficient conditions for a submodule of a QTAG-module to be large and characterize them. Also, we investigate some properties of large submodules shared by $\Sigma$-modules, summable modules, $\sigma$-summable modules, and so on.
\end{abstract}

\section{Introduction and Preliminaries}

All the rings $R$ considered here are associative with unity and modules $M$ are unital QTAG-modules. An element $x \in M$ is uniform, if $x R$ is a nonzero uniform (hence uniserial) module and, for any $R$-module $M$ with a unique composition series, $d(M)$ denotes its composition length. For a uniform element $x \in M, e(x)=d(x R)$ and $H_{M}(x)=\sup \{d(y R / x R) \mid y \epsilon$ $M, x \in y R$ and $y$ uniform $\}$ are the exponent and height of $x$ in $M$, respectively. $H_{k}(M)$ denotes the submodule of $M$ generated by the elements of height at least $k$ and $H^{k}(M)$ is the submodule of $M$ generated by the elements of exponents at most $k$. For any arbitrary $x \in M, H(x)=k$ if $x \in H_{k}(M)$ but $x \notin H_{k+1}(M) . M$ is $h$-divisible if $M=M^{1}=\bigcap_{k=0}^{\infty} H_{k}(M)$ and it is $h$-reduced if it does not contain any $h$-divisible submodule. In other words it is free from the elements of infinite height.

A submodule $N$ of $M$ is $h$-pure in $M$ if $N \cap H_{k}(M)=$ $H_{k}(N)$, for every integer $k \geq 0$. For a limit ordinal $\alpha$, $H_{\alpha}(M)=\bigcap_{\rho<\alpha} H_{\rho}(M)$, for all ordinals $\rho<\alpha$, and it is $\alpha$ pure in $M$ if $H_{\sigma}(N)=H_{\sigma}(M) \cap N$ for all ordinals $\sigma<$ $\alpha$ and it is an isotype if it is $\alpha$-pure for every ordinal $\alpha$. A submodule $B \subseteq M$ is a basic submodule of $M$, if $B$ is $h$-pure in $M, B=\bigoplus B_{i}$, where each $B_{i}$ is the direct sum of uniserial modules of length $i$ and $M / B$ is $h$-divisible. For a QTAG-module $M$, the $\sigma$ th-Ulm invariant of $M, f_{M}(\sigma)$ is the cardinal number $g\left(\operatorname{Soc}\left(H_{\sigma}(M)\right) / \operatorname{Soc}\left(H_{\sigma+1}(M)\right)\right)$ [1]. Several results which hold for TAG-modules also hold good for QTAG-modules [2].

A module $M$ is summable if $\operatorname{Soc}(M)=\bigoplus_{\tau<\alpha} S_{\alpha}$, where $S_{\alpha}$ is the set of all elements of $H_{\alpha}(M)$ which are not in $H_{\alpha+1}(M)$, where $\tau$ is the length of $M$. A QTAG-module $M$ is called $\sigma$-summable if $\operatorname{Soc}(M)=\bigcup_{n<\omega} M_{n}, M_{n} \subseteq M_{n+1}$ and, for every positive integer $n$, there is an ordinal $\alpha_{n}$ such that $M_{n} \cap H_{\alpha_{n}}(M)=0, \alpha_{n}$ < length of $M$.

For any uniform element $x \in M$, there exist uniform elements $x_{1}, x_{2}, \ldots$ such that $x R \supseteq x_{1} R \supseteq x_{2} R \supseteq \cdots$ and $d\left(x_{i} R / x_{i+1} R\right)=1$. Now the Ulm-sequence of $x$ is defined as $U(x)=\left(H(x), H\left(x_{1}\right), H\left(x_{2}\right), \ldots\right)$. $U$ sequences are defined as $U(x)$. This is analogous to the $U l m$-sequences defined in groups [3]. These sequences are partially ordered because $U(x) \leq U(y)$ if $H\left(x_{i}\right) \leq H\left(y_{i}\right)$ for every $i$. For the sequence $n=\left(n_{0}, n_{1}, n_{2}, \ldots\right)$ of nonnegative, nondecreasing integers we may consider $L$ as the submodule of $M$ generated by the elements $x$ of $M$ for which $U(x) \geq n$. If $f$ is an endomorphism of $M$, then $H(x) \leq H(f(x))$, and therefore 
$L$ is fully invariant. Therefore with every large submodule $L$ of $M$ we may associate a sequence $n(L)$.

\section{Some Characterizations of Large Submodules}

In this section we study and characterize the properties of fully invariant and large submodules of QTAG-modules. We also discuss the properties of large submodules inherited from the containing module.

We start with the facts which are true for any module. For a fully invariant submodule $N$ of a QTAG-module $M$ and an endomorphism $f$ of $M$, it induces an endomorphism $\bar{f}$ of $M / N$ such that $\bar{f}(x+N)=f(x)+N$. On the other hand for the endomorphism $\bar{f}$ of $M / N$ induced by an endomorphism $f$ of $M$ and a fully invariant submodule $K / N \subseteq M / N, \bar{f}(x+$ $N)=f(x)+N \in K / N$. That is, $f(x) \in K$ and $K$ is fully invariant in $M$. For a fully invariant submodule $A \subseteq M=$ $\bigoplus M_{i}, A=\bigoplus\left(A \cap M_{i}\right)$ and each $A \cap M_{i}$ is fully invariant in $M_{i}$.

For any sequence $n=\left(n_{1}, n_{2}, \ldots\right)$ we define $M(n)$ as the submodule of $M$, generated by the elements $x$ for which $U(x) \geq n$. This submodule is a large submodule of $M$. In fact for every large submodule there is a sequence and, for every sequence, there is a large submodule [4].

For a QTAG-module $M$, consider the homomorphism $f$ : $M \rightarrow M / M^{1}$. As $M^{1}=\bigcap_{k=0}^{\infty} H_{k}(M), f$ is height preserving. This implies that $H(x)=H(f(x))$ and $U(x)=U(f(x))$ for all $x \in M$.

We conclude that $L / M^{1}$ is large in $M / M^{1}$ if and only if $L$ is large in $M$. In a module $M$ without elements of infinite height, consider a fully invariant submodule $K$ of $M$, and $x \in$ $\operatorname{Soc}(K)$ such that $n=H(x) \leq H(y)$ for every $y \in \operatorname{Soc}(K)$. Let $z \in \operatorname{Soc}(M)$, such that $H(z) \geq n$. Then there exists an endomorphism $f$ of $M$ such that $f(x)=z$; therefore $z \in K$ and $\operatorname{Soc}(K)=\operatorname{Soc}\left(H_{n}(M)\right)$.

Remark 1. For any large submodule $L$ of $M$, $\operatorname{Soc}(L)=$ $\operatorname{Soc}\left(H_{n}(M)\right)$ for some positive integer $n$.

Lemma 2. Let $N$ be submodule of $M$ such that $\operatorname{Soc}\left(H_{n_{k}}(M)\right) \subseteq$ $\operatorname{Soc}\left(H_{k}(N)\right)$ for $k=0,1,2, \ldots$, where the sequence of positive integers $n_{0}, n_{1}, n_{2}, \ldots$ is monotonically increasing. Then $M=$ $N+B$ for any basic submodule $B$ of $M$.

Proof. Let $B=\bigoplus B_{i}$ be a basic submodule of $M$ and $M=$ $B_{1} \oplus \cdots \oplus B_{k} \oplus\left(B_{k}^{*}, H_{k}(M)\right)[5]$. Then

$$
\begin{aligned}
\operatorname{Soc}(M) & =\operatorname{Soc}\left(B_{1}+\cdots+B_{k}\right) \oplus \operatorname{Soc}\left(H_{k}(M)\right) \\
& =\operatorname{Soc}\left(B_{1} \oplus \cdots \oplus B_{n_{k}}\right) \oplus \operatorname{Soc}\left(H_{k}(N)\right) .
\end{aligned}
$$

Now suppose, for every $x \in M, e(x) \leq k$ implies that $x \in(B+N)$. Consider $x \in M$ such that $e(x)=k+1$; then there exists $y \in M$ such that $d(x R / y R)=k$. Now $y \in \operatorname{Soc}(M)$ and $y=b+z$, where $b \in B$ and $z \in \operatorname{Soc}\left(H_{k}(N)\right)$, ensuring the existence of $z^{\prime}$ such that $d\left(z^{\prime} R / z R\right)=k$. By the $h$-purity of $B$, there exists $b^{\prime} \in B$, such that $d\left(b^{\prime} R / b R\right)=k$. Now $e\left(x-b^{\prime}-\right.$ $\left.z^{\prime}\right) \leq k$, and thus $x-b^{\prime}-z^{\prime} \in B+N$ or $x \in B+N$ implying that $M=B+N$.

The following remarks are significant to be stated.

Remark 3. Let $L$ be a large submodule of an unbounded QTAG-module $M$ without elements of infinite height $M$ and $B$ a proper basic submodule of $M$. Then

$$
\frac{M}{B}=\frac{(B+L)}{B} \cong \frac{L}{(B \cap L)} ;
$$

therefore $L$ is unbounded. Conversely for an unbounded fully invariant submodule $L$ of $M, H_{k}(L)$ is fully invariant for all $k \in \mathbb{Z}^{+}$. As an immediate consequence of Lemma $2, L$ is a large submodule of $M$. We can say that the unbounded fully invariant submodules of $M$ are exactly the large submodules of $M$.

Remark 4. If $B_{i}$ is the direct sum of uniserial modules of length $i$ and $x(\neq 0) \in B_{i}$, then

$$
U(x)=\left\langle n_{0}, n_{1}, \ldots, n_{i-n_{0}-1}, \ldots, \infty\right\rangle,
$$

where $n_{0}=H(x)$ and $n_{k}=n_{0}+k, 0 \leq k \leq i-n_{0}-1$.

Remark 5. Let $B_{i}$ be the direct sum of uniserial modules of length $i$ and $x, y \in B_{i}$. Then there exists an endomorphism $f$ of $B_{i}$ with $f(x)=y$, if and only if $H(x) \leq H(y)$.

Remark 6. Let $A$ be a fully invariant submodule of $B_{i}$, a direct sum of uniserial modules of length $i$. Then $A=H_{n_{i}}\left(B_{i}\right)$, where $n_{i} \leq i$. If $A=0$, if $n_{i}=i$, and if $A \neq 0$, then $n_{i}=\min \{H(x), x \in A\}$.

Remark 7. If $B_{i}$ and $B_{i+j}$ are the direct sums of uniserial modules of length $i$ and $i+j$, respectively, and $x \in B_{i}, y \in B_{i+j}$, then

(i) there exists a homomorphism $f: B_{i} \rightarrow B_{i+j}$ such that $f(x)=y$ if and only if $e(x) \geq e(y)$,

(ii) there exists a homomorphism $g: B_{i+j} \rightarrow B_{i}$ such that $g(y)=x$ if and only if $H(x) \geq H(y)$.

Theorem 8. Let $B=\bigoplus_{i} B_{i}$, where each $B_{i}$ is the direct sum of uniserial modules of length $i$. Then $L$ is a fully invariant submodule of $B$ if and only if $L=\bigoplus_{i} H_{n_{i}}\left(B_{i}\right)$, where $n_{i} \leq i$, for every $i \in \mathbb{Z}^{+}$and $n_{i} \leq n_{i+j} \leq n_{i}+j$ for $i, j \in \mathbb{Z}^{+}$. A fully invariant submodule $L$ is large in $B$ if and only if $L=\bigoplus_{i} H_{n_{i}}\left(B_{i}\right)$; the above conditions hold and the sequence $\left\langle 1-n_{1}, 2-n_{2}, 3-n_{3}, \ldots\right\rangle$ is unbounded if $B$ is unbounded.

Proof. Let $L$ be a fully invariant submodule of $B$. Then

$$
L=L \cap B=\bigoplus\left(B_{i} \cap L\right)=\bigoplus H_{n_{i}}\left(B_{i}\right)
$$

by the facts mentioned above and Remark 6 . Now $n_{i} \leq i$ for $i \in \mathbb{Z}^{+}$and the first condition holds. If $L=0$, then $H_{n_{i}}\left(B_{i}\right)=0$ for every $i$; therefore $n_{i}=i$ for every $i$ and the second condition holds. If $L \neq 0$, then there exists a least positive integer $k$ such that $H_{n_{k}}\left(B_{k}\right) \neq 0$. Then $H_{n_{i}}\left(B_{i}\right) \neq 0$ for 
all $i \geq k$, where $B_{i} \neq 0$. Since $\operatorname{Soc}\left(B_{k}\right)=\operatorname{Soc}\left(H_{k-1}\left(B_{k}\right)\right) \subseteq L$, this implies that $\operatorname{Soc}\left(H_{k-1}(B)\right) \subseteq \operatorname{Soc}(L)$. Again $\operatorname{Soc}\left(B_{i}\right)=$ $\operatorname{Soc}\left(H_{k-1}\left(B_{i}\right)\right)$ for $i \geq k$; we have $\operatorname{Soc}\left(B_{i}\right) \subseteq L \cap B_{i}=H_{n_{i}}\left(B_{i}\right)$. Now suppose $L \neq 0$ and $B_{i} \neq 0 \neq B_{i+j}$. If $H_{n_{i+j}}\left(B_{i+j}\right)=0$, then $H_{n_{i}}\left(B_{i}\right)=0$ and $n_{i}=i, n_{i+j}=i+j=n_{i}+j$ and the second condition holds. We assume that $H_{n_{i+j}}\left(B_{i+j}\right) \neq 0$. Consider $x \in B_{i}$ such that $H(x) \geq n_{i+j}$ and $y \in H_{n_{i+j}}\left(B_{i+j}\right)$ such that $H(y)=n_{i+j}$. Now, by Remark 7, there exists an endomorphism $g$ of $B$ mapping $y$ onto $x$. Hence $x \in L$ and $H_{n_{i+j}}\left(B_{i}\right) \subseteq L \cap B_{i}=H_{n_{i}}\left(B_{i}\right)$; thus $n_{i} \leq n_{i+j}$.

Now suppose $H_{n_{i}}\left(B_{i}\right)=0$. Then $n_{i}=i$ so $n_{i+j} \leq i+j=$ $n_{i}+j$. If $H_{n_{i}}\left(B_{i}\right) \neq 0$ and $y \in B_{i+j}$ such that $H(y) \geq n_{i}+j$, we may choose $x \in B_{i}$ such that $H(x)=n_{i}$. Then $e(x)=i-n_{i}$ and $e(y) \leq i+j-\left(n_{i}+j\right)=i-n_{i}$. By Remark 7, there exists an endomorphism $f$ of $B$ with $f(x)=y$. Thus $y \in L$ and we have $H_{n_{i+j}}\left(B_{i+j}\right) \subseteq L \cap B_{i+j}=H_{n_{i+j}}\left(B_{i+j}\right)$; therefore $n_{i+j} \leq n_{i}+j$.

If $B_{i} \neq 0 \neq B_{i+j}$, then $n_{i} \leq n_{i+j} \leq n_{i}+j$ but if $B_{i}=0$, we may define $n_{i}$ so that this inequality holds for all $i$. Thus all fully invariant submodules of $B$ are the direct sums of $H_{n_{i}}\left(B_{i}\right)$. If $L$ is a large submodule of $B$ and $B$ is unbounded, then, by Lemma $2, L$ is also unbounded. Therefore $\left\langle 1-n_{1}, 2-n_{2}, 3-\right.$ $\left.n_{3}, \ldots\right\rangle$ must be unbounded.

For the converse, suppose $L=\bigoplus H_{n_{i}}\left(B_{i}\right)$, where $n_{i} \leq i$ for all $i \in \mathbb{Z}^{+}$and $n_{i} \leq n_{i+j} \leq n_{i}+j$ for all $i, j \in \mathbb{Z}^{+}$. To establish the full invariance of $L$, we consider any $i \in \mathbb{Z}^{+}$and $x \in H_{n_{i}}\left(B_{i}\right)$. We have to show that for any endomorphism $f$ of $B, f(x) \in L$. Consider $x \neq 0$, such that $f(x)=x_{1}+\cdots+x_{l}$, where $x_{r} \in B_{r}$ and $H(x) \leq H(f(x))=\min \left(H\left(x_{k}\right)\right), 1 \leq$ $k \leq l, e(x) \geq e(f(x))=\max \left\{e\left(x_{k}\right) \mid 1 \leq k \leq l\right\}$. If $k \leq i$, then $H\left(x_{k}\right) \geq H(x) \geq n_{i}$ so $x_{k} \in H_{n_{i}}\left(B_{k}\right) \subseteq H_{n_{k}}\left(B_{k}\right)$, because $n_{k} \leq n_{i}$; hence $x_{k} \in L$. If $k=i+j$, then $e\left(x_{k}\right) \leq e(x) \leq i-n_{i}=$ $i+j-\left(n_{i}+j\right) \leq i+j-n_{i+j}$ because $n_{i+k} \leq n_{i}+k$. Thus $x_{k} \in H_{i+j-n_{i+j}}\left(B_{i+j}\right)=H_{n_{i+j}}\left(B_{i+j}\right) \subseteq L$.

This implies that $L$ is a fully invariant submodule of $B$. If $B$ is unbounded and $\left\langle 1-n_{1}, 2-n_{2}, 3-n_{3}, \ldots\right\rangle$ is also unbounded, then $L$ is unbounded and is therefore a large submodule of $B$ by Remark 3 .

Corollary 9. If $L$ is a large submodule of a $Q T A G$-module $M$, then $M / L$ is a direct sum of uniserial modules.

Proof. For any basic submodule $B$ of $M$,

$$
\begin{aligned}
\frac{M}{L} & =\frac{(B+L)}{L} \cong \frac{B}{(B \cap L)}=\frac{\bigoplus_{i} B_{i}}{\bigoplus_{i} H_{n_{i}}\left(B_{i}\right)} \\
& \cong \bigoplus_{i}\left(\frac{B_{i}}{H_{n_{i}}\left(B_{i}\right)}\right)
\end{aligned}
$$

and the result follows.

Corollary 10. For any large submodule $L$ of $M, L^{1}=M^{1}$.

Proof. Since $M / L$ is a direct sum of uniserial modules, $(M / L)^{1}=0$ or $M^{1}=L^{1}$.

Theorem 11. Let $N$ be h-pure submodule of a QTAG-module $M$ and $L$ a large submodule of $N$. Then there exists a large submodule $L^{\prime}$ of $M$ such that $L^{\prime} \cap N=L$. If $M / N$ is h-divisible, then $L^{\prime}$ is the closure of $L$ in $M$ and is therefore uniquely determined by $L$ and $M / L^{\prime} \cong N / L$.

Proof. Let $L=N(n)$ and $L^{\prime}=M(n)$. Since $N$ is $h$-pure in $M$ and $n=\left\langle n_{1}, n_{2}, \ldots\right\rangle$ is a $U$-sequence for $N$, we have that $n$ is a $U$-sequence for $M$. Thus $L^{\prime}$ is a large submodule of $M$.

If $x \in L$, then $U_{M}(x)=U_{N}(x) \geq n$; therefore $x \in L^{\prime} \cap N$ and $L \subseteq L^{\prime} \cap N$. Conversely if $y \in L^{\prime} \cap N$, then $U_{N}(y)=$ $U_{M}(y) \geq n$ implies that $y \in L$ or $L^{\prime} \cap N \subseteq L$. Thus $L=L^{\prime} \cap N$.

Let $M / N$ be $h$-divisible and $L^{\prime}$ a large submodule of $M$ with $L^{\prime} \cap N=L$. Then

$$
\frac{L^{\prime}}{L}=\frac{L^{\prime}}{\left(L^{\prime} \cap N\right)} \cong \frac{\left(N+L^{\prime}\right)}{N}=\frac{M}{N} .
$$

That is, $L^{\prime} / L$ is $h$-divisible. But $M / L^{\prime} \cong(M / L) /\left(L^{\prime} / L\right)$, where $L^{\prime} / L$ is a direct summand of $M / L$; we have $M / L \cong\left(L^{\prime} / L\right) \oplus$ $\left(M / L^{\prime}\right)$ and $M / L^{\prime}$ is a direct sum of uniserial modules [6]. Now $M / L^{\prime} \cong N / L$, thus

$$
\frac{M}{L^{\prime}}=\frac{\left(N+L^{\prime}\right)}{L^{\prime}} \cong \frac{N}{\left(N \cap L^{\prime}\right)}=\frac{N}{L} .
$$

Now we characterize large submodules in terms of Ulm invariants.

Theorem 12. Let $L$ be a submodule of a QTAG-module $M$. Then $L$ is a large submodule of $M$ if and only if $L=$ $\sum_{k=1}^{\infty} H_{n_{k}}\left(H^{k-n_{k}}(M)\right)$, where

(i) $n_{k} \leq k, k \in \mathbb{Z}^{+}$,

(ii) $n_{k} \leq n_{k+1} \leq n_{k}+1$,

(iii) the sequence $\left\langle 1-n_{1}, 2-n_{2}, 3-n_{3}, \ldots\right\rangle$ is unbounded if $M$ is unbounded and the Ulm-invariants of $L$ are given by $f_{L}(n)=\sum_{k}\left(f_{M}(k-1)\right), k-n_{k}-1=n$, for all $n \in \mathbb{Z}^{+}$

Proof. Suppose $L=\sum_{k=1}^{\infty} H_{n_{k}}\left(H^{k-n_{k}}(M)\right)$. Since $H_{n_{k}}\left(H^{k-n_{k}}(M)\right)$ 's are fully invariant submodules, their sum is again fully invariant submodule of $M$. If $M$ is bounded, then $L$ is large. If $M$ is unbounded, then, by the third condition, for each $j \in \mathbb{Z}^{+}$, there exists a positive integer $i$ such that $i-n_{i}>j$ or $i>n_{i}+j$.

Since, $i>n_{i}+j$ and $\operatorname{Soc}\left(H_{i}(M)\right) \subseteq \operatorname{Soc}\left(H_{j+n_{i}}(M)\right) \subseteq$ $H_{j}(L)$, If $x \in \operatorname{Soc}\left(H_{j+n_{i}}(M)\right)$, there exists $y \in M$ such that $d(y R / x R)=j+n_{i}$, where $e(x)=1$. Now $y \in H^{j+n_{i}+1}(M)$, where $i-n_{i}>j$ or $i-n_{i} \geq j+1$; thus $i \geq n_{i}+j+1$ and $y \in H^{i}(M)$.

If $d(y R / z R)=n_{i}$, then $z \in H_{n_{i}}\left(H^{i-n_{i}}(M)\right) \subseteq L$ and $x \in$ $H_{j}(L)$ because $d(y R / x R)=j+n_{i}$ and $d(z R / x R)=j$. Now, by Lemma $2, L+B=M$, for every basic submodule $B$ of $M$, and $L$ is a large submodule of $M$.

Conversely suppose $L$ is a large submodule of $M$. Then for any basic submodule $B$ of $M, L \cap B$ is a large submodule of $B$ and, by Theorem $8, L \cap B=\bigoplus_{k} H_{n_{k}}\left(B_{k}\right)$, where $k \in \mathbb{Z}^{+}$and $n_{k}$ 's satisfy the given conditions. 
Now,

$$
\begin{aligned}
H_{n_{j}}\left(B_{j}\right)=H_{n_{j}}\left(H^{j-n_{j}}\left(B_{j}\right)\right) \subseteq H_{n_{j}}\left(H^{j-n_{j}}(B)\right), & \\
& \text { for every } j \in \mathbb{Z}^{+}
\end{aligned}
$$

and, for each $j \in \mathbb{Z}^{+}$,

$$
H_{n_{j}}\left(H^{j-n_{j}}(B)\right) \subseteq \Sigma H_{n_{k}}\left(H^{k-n_{k}}(B)\right) .
$$

This implies that $\bigoplus H_{n_{k}}\left(B_{k}\right) \subseteq \sum H_{n_{k}}\left(H^{k-n_{k}}(B)\right)$.

For the converse, consider $x \in H_{n_{j}}\left(H^{j-n_{j}}(B)\right)$, where $x=$ $x_{1}+x_{2}+\cdots+x_{m}, x_{i} \in B_{i}$. Then $H\left(x_{i}\right) \geq H(x) \geq n_{j}$ for $x_{i}, 1 \leq i \leq m$ and $e\left(x_{i}\right) \leq e(x) \leq j-n_{i}$ for all $x_{i}^{\prime}$ s. Now, for $i \leq j$, we have $H\left(x_{i}\right) \geq n_{j} \geq n_{i}$ and $x_{i} \in H_{n_{i}}\left(B_{i}\right)$.

If $i=j+l$ for $l \in \mathbb{Z}^{+}$, then $e\left(x_{i}\right) \leq j-n_{j}=j+l-\left(n_{j}+l\right) \leq$ $j+l-n_{j+l}$. (by the given condition). Therefore

$$
x_{i} \in\left(H^{j+l-\left(n_{j+l}\right)}\left(B_{j+l}\right)\right)=H_{n_{j+l}}\left(B_{j+l}\right)=H_{n_{i}}\left(B_{i}\right)
$$

and $H_{n_{j}}\left(H^{j-n_{j}}(B)\right) \subseteq \bigoplus_{k} H_{n_{k}}\left(B_{k}\right)$. Let $L^{\prime}=$ $\sum H_{n_{k}}\left(H^{k-n_{k}}(M)\right)$. Now $L^{\prime} \cap B=\sum H_{n_{k}}\left(H^{k-n_{k}}(B)\right)=L \cap B$. Since $B$ is $h$-pure in $M$ and $M / B$ is $h$-divisible, $L^{\prime}=L$, by Theorem 11. Again $L \cap B$ is a basic submodule of $L$; thus $f_{L}(n)=f_{L \cap B}(n)$, for all $n \in \mathbb{Z}^{+}$.

If $L \cap B=\bigoplus\left(L \cap B_{i}\right)_{i}$, where $(L \cap B)_{i}$ is the direct sum of uniserial modules of length $i$, then $f_{(L \cap B)}(n)=g\left((L \cap B)_{n+1}\right)=$ $g\left(\bigoplus H_{n_{k}}\left(B_{k}\right)\right)$, where $H_{n_{k}}\left(B_{k}\right)$ is a direct sum of uniserial modules of length $n+1$.

Again,

$$
\begin{aligned}
f_{L \cap B}(n) & =g\left(\bigoplus_{k} H_{n_{k}}\left(B_{k}\right)\right)=\sum_{k}\left(g\left(H_{n_{k}}\left(B_{k}\right)\right)\right) \\
& =\sum_{k}\left(g\left(B_{k}\right)\right)=\sum_{k}\left(f_{B}(k-1)\right) \\
& =\sum_{k}\left(f_{M}(k-1)\right), \quad \text { where } k-n_{k}-1=n .
\end{aligned}
$$

And the proof is complete.

\section{Properties of Large Submodules of QTAG-Modules}

In this section we compare the structures of QTAG-modules and their large submodules. We investigate the characteristics of QTAG-modules which are preserved by their large submodules. We start with the $\sum$-modules, that is, the modules whose high submodules are direct sums of uniserial modules [7]. Then we study summable, $\sigma$-summable, $(\omega+1)$ projective, and $h$-pure complete QTAG-modules.

Singh [8] proved that a QTAG-module $M$ is a direct sum of uniserial submodules if and only if $M$ is the union of an ascending sequence of submodules $M_{n}, n=1,2,3, \ldots$, such that, for every $n$, there exists $k_{n}>0$ and $H_{M}(x) \leq k_{n}$ for all $x \in M_{n}$.

This helps us to prove the following.
Theorem 13. A QTAG-module is a $\sum$-module if and only if $\operatorname{Soc}(M)=\bigcup_{k=1}^{\infty} M_{k}$, where $M_{k} \subset M_{k+1}$ and for every $k \in$ $N, M_{k} \cap H_{k}(M)=\operatorname{Soc}\left(M^{1}\right)$.

Proof. Since $M$ is a $\sum$-module, it contains a high submodule $N$ such that $N$ is a direct sum of uniserial modules.

Again $N$ is a high submodule [9] of $M$ if and only if $N$ is $h$-pure in $M$ and $\operatorname{Soc}(M)=\operatorname{Soc}(N)+\operatorname{Soc}\left(M^{1}\right)$. Therefore by the above result [8], $\operatorname{Soc}(N)=\bigcup_{k=1}^{\infty} N_{k}, N_{k} \subseteq N_{k+1}$, and $N_{k} \cap$ $H_{k}(N)=0$, and we deduce $\operatorname{Soc}(M)=\bigcup_{k=1}^{\infty}\left(N_{k}+\operatorname{Soc}\left(M^{1}\right)\right)$. If we put $M_{k}=\operatorname{Soc}\left(M^{1}\right)+N_{k}$, then $M_{k} \subseteq M_{k+1}$ and $\left(\operatorname{Soc}\left(M^{1}\right)+\right.$ $\left.N_{k}\right) \cap \operatorname{Soc}\left(M^{1}\right)+\operatorname{Soc}\left(H_{k}(N)\right)=\operatorname{Soc}\left(M^{1}\right)+\left(N_{k} \cap H_{k}(N)\right)=$ Soc $\left(M^{1}\right)$, because $H_{k}(N)$ is a high submodule of $H_{k}(M)$.

For the converse if $\operatorname{Soc}(N)=\bigcup_{k=1}^{\infty}\left(M_{k} \cap N\right)=\bigcup_{k=1}^{\infty} N_{k}$, where we put $N_{k}=N \cap M_{k}$, then $N_{k} \subseteq N_{k+1}$. Also

$$
\begin{aligned}
N_{k} \cap H_{k}(N) & =M_{k} \cap H_{k}(M)=M_{k} \cap\left(H_{k}(M) \cap N\right) \\
& =M_{k} \cap H_{k}(M) \cap N=M^{1} \cap N=0 .
\end{aligned}
$$

Therefore $N$ is a direct sum of uniserial modules and $M$ is a $\Sigma$-module.

Now we may prove the following.

Theorem 14. A QTAG-module $M$ is a $\Sigma$-module if and only if its large submodule $L$ is a $\sum$-module.

Proof. Since $L^{1}=M^{1}$ [6], there is a natural number $m$ such that $\operatorname{Soc}(L)=\operatorname{Soc}\left(H_{m}(M)\right)$ and $\operatorname{Soc}\left(H_{n}(L)\right)=$ $\operatorname{Soc}\left(H_{t_{n}}(M)\right)$ for every $n<\omega$ and some $t_{n}$ such that $m \leq$ $t_{n}<\omega$. If $M$ is a $\Sigma$-module, then, by Theorem $13, \operatorname{Soc}(M)$ is the union of ascending chain of submodules $M_{k}$ such that $M_{k} \subseteq M_{k+1}$ and $M_{k} \cap H_{k}(M)=\operatorname{Soc}\left(M^{1}\right)$ for every $k \in N$.

This implies that $\operatorname{Soc}(L)=\bigcup_{k<\omega}\left(M_{k} \cap L\right)$ and $M_{k} \cap L \subseteq$ $M_{k+1} \cap L$. Therefore

$$
\begin{aligned}
M_{k} \cap L \cap H_{k}(L) & =M_{k} \cap H_{k}(L) \subseteq M_{k} \cap H_{k}(M) \\
& =\operatorname{Soc}\left(M^{1}\right)=\operatorname{Soc}\left(L^{1}\right) .
\end{aligned}
$$

Now Theorem 13 indicates that $L$ is a $\Sigma$-module. Conversely suppose $L$ is a $\sum$-module. Therefore

$$
\begin{aligned}
& \operatorname{Soc}(L)=\bigcup_{n<\omega} L_{n}, \\
& \qquad L_{n} \subseteq L_{n+1}, L_{n} \cap H_{n}(L)=\operatorname{Soc}\left(L^{1}\right) .
\end{aligned}
$$

$\operatorname{Again} \operatorname{Soc}\left(H_{m}(M)\right)=\bigcup_{n<\omega} L_{n}$. Now

$$
\begin{aligned}
L_{n} \cap \operatorname{Soc}\left(H_{t_{n}}(M)\right) & =L_{n} \cap \operatorname{Soc}\left(H_{n}(L)\right)=\operatorname{Soc}\left(L^{1}\right) \\
& =\operatorname{Soc}\left(M^{1}\right) .
\end{aligned}
$$

Thus, by Theorem $13, H_{m}(M)$ is a $\sum$-module, and so is $M$.

To study the other relations between a module $M$ and its large submodule $L$ we need the following lemma. 
Lemma 15. Isotype submodules of countable length of summable QTAG-modules are again summable.

Proof. Let $N$ be an isotype submodule of countable length $\rho$ in the summable module $M$. Now there is a $H_{\rho}(M)$-high submodule $K$ of $M$ such that $N \subseteq K$. Since $\operatorname{Soc}(M)=$ $\bigoplus_{\sigma<\rho} S_{\sigma}$, there is $H_{\rho}(M)$-high submodule $P$ of $M$ such that $\operatorname{Soc}(P)=\bigoplus_{\sigma<\rho} S_{\sigma}$.

Again, for every ordinal $\rho$, every $H_{\rho}(M)$-high submodule is isotype; therefore $P$ is isotype and it is summable. The socles of $H_{\rho}(M)$-high submodules have the same images under the canonical map $M \rightarrow M / H_{\rho}(M)$ because this maps $H_{\rho}(M)$-high submodules isomorphically in a height preserving manner onto submodules of $M / H_{\rho}(M)$.

Now $N$ is isotype in a summable module $K$ of countable length $\rho$. Therefore $\operatorname{Soc}(K)$ is the union of an ascending chain of submodules $K_{n}$, where for every $n$ the heights of elements of $K_{n}$ assume but a finite numbers of values.

Now $\operatorname{Soc}(N)=\cup K_{n} \cap N, n=1,2,3, \ldots$, and the heights of the elements of $K_{n} \cap N$ assume a finite numbers of different values. Thus $N$ is summable.

The following result shows that summability is shared by large submodules.

Theorem 16. Let $L$ be a large submodule of a QTAG-module $M$. Then $M$ is summable if and only if $L$ is summable.

Proof. Suppose $M$ is summable; that is, $\operatorname{Soc}(M)=\bigoplus_{\beta<\alpha} M_{\beta}$, where the nonzero elements of $M_{\beta}$ 's are contained in $H_{\beta}(M)$ but they do not belong to $H_{\beta+1}(M)$, for every $\beta<\alpha$.

Again $L$ is fully invariant submodule of $M$ and $H_{\rho}(M)=$ $H_{\rho}(L)$ for all ordinals $\rho \geq \omega$, $\operatorname{Soc}(L)=\bigoplus_{\beta<\alpha}\left(M_{\beta} \cap L\right)$, where the nonzero elements of $M_{\beta} \cap L$ are contained in $H_{\beta}(L)$ and not contained in $H_{\beta+1}(L)$ for every $\omega \leq \beta<\alpha$. Since $\operatorname{Soc}\left(H_{n}(L)\right)=\operatorname{Soc}\left(H_{t_{n}}(M)\right)$, whenever $1 \leq n<\omega, n \leq$ $t_{n}<\omega, M_{\beta} \cap L \subseteq L$, but $\left(M_{\beta} \cap L\right) \cap H_{1}(L)=0$, for each $\beta<t_{1}$. By transfinite induction $M_{\beta} \cap L \subseteq H_{1}(L)$ and $\left(M_{\beta} \cap L\right) \cap H_{2}(L)=0$, for $t_{1} \leq \beta<t_{2}$ and so on; that is, $H_{\beta} \cap L \subseteq H_{n}(L)$ and $\left(H_{\beta} \cap L\right) \cap H_{n+1}(L)=0$, for $t_{n} \leq \beta<t_{n+1}$.

If we put $L_{0}=\bigoplus_{0 \leq \beta<t_{1}}\left(M_{\beta} \cap L\right), L_{1}=\bigoplus_{t_{1} \leq \beta<t_{2}}\left(M_{\beta} \cap\right.$ $L), \ldots$, and $L_{n}=\bigoplus_{t_{n} \leq \beta<t_{n+1}}\left(M_{\beta} \cap L\right)$, where $n<\omega$, and $M_{\beta} \cap L=L_{\beta}$ if $\beta \geq \omega$, then $\operatorname{Soc}(L)=\bigoplus_{\beta<\alpha} L_{\beta}$ if $L_{\beta} \subseteq$ $H_{\beta}(L)$ and $L_{\beta} \cap H_{\beta+1}(L)=0$. Therefore $L$ is summable.

Conversely suppose $L$ is summable. So, $L^{1}=M^{1}$ is summable as its fully invariant submodule. Moreover, by Lemma $15, L$ being summable implies that $L$ is a $\Sigma$-module. Now by Theorem 14, $M$ is also a $\Sigma$-module. For a high submodule $N$ of $M$, $\operatorname{Soc}(N) \oplus \operatorname{Soc}\left(M^{1}\right)=\operatorname{Soc}(M)$.

Since $N$ is a direct sum of uniserial modules, $\operatorname{Soc}(N)=$ $\bigoplus_{k<\omega} N_{k}$, where $N_{k} \subseteq H_{k}(M)$ and $N_{k} \cap H_{k+1}(M)=0$ because $N$ is $h$-pure in $M$. Again the summability of $M^{1}$ ensures that $\operatorname{Soc}\left(M^{1}\right)=\bigoplus_{\beta<\alpha} K_{\beta}$, where $K_{\beta} \subseteq H_{\beta}\left(M^{1}\right)$ and $K_{\beta} \cap H_{\beta+1}\left(M^{1}\right)=0$. Therefore, $K_{\beta} \subseteq H_{\omega+\beta}(M)$ and $K_{\beta} \cap H_{\omega+\beta+1}(M)=0$. This implies that

$$
\operatorname{Soc}(M)=\bigoplus_{k<\omega} N_{k} \bigoplus_{\omega \leq \omega+\beta<\omega+\alpha} K_{\beta} .
$$

We may infer now that $M$ is summable.
Theorem 17. Let $L$ be the large submodule of $M$. Then $M$ is $\sigma$-summable if and only if $L$ is $\sigma$-summable.

Proof. Suppose $M$ is unbounded. Then length of $M=$ length of $L \geq \omega$. If $M$ is $\sigma$-summable, then $L$ is also $\sigma$-summable being a submodule of equal length.

If $M$ is bounded the result holds trivially.

Conversely suppose $L$ is $\sigma$-summable. Therefore $\operatorname{Soc}(L)=$ $\bigcup_{n<\omega} L_{n}, L_{n} \subseteq L_{n+1}$ and $L_{n} \cap H_{\alpha_{n}}(L)=0$ for all $n \geq 0$ and some $\alpha_{n}<$ length of $M$.

Now, $\operatorname{Soc}\left(H_{m}(M)\right)=\bigcup_{n<\omega} L_{n}$. Since $L^{1}=M^{1}[6]$, $H_{\alpha}(M)=H_{\alpha}(L)$ for each ordinal $\alpha \geq \omega$ and $\operatorname{Soc}\left(H_{\alpha_{n}}(L)\right)=$ Soc $\left(H_{k_{n}}(M)\right)$ for $\alpha_{n}<\omega$ and some $k_{n} \geq \max \left(\alpha_{n}, m\right)$ because $H_{\alpha_{n}}(L)$ is large in $M$ and $H_{\alpha_{n}}(M)$ both. Thus $L_{n} \cap H_{s_{n}}(M)=$ $L_{n} \cap H_{\alpha_{n}}(L)=0$, whenever $s_{n}$ < length of $M=$ length of $H_{m}(M) \geq \omega, s_{n}=\alpha_{n} \geq \omega$ or $\omega>s_{n}=k_{n}$.

We may define $M_{n}=\left\{x \mid x \in \operatorname{Soc}(M) \cap L_{n}\right.$ and $x \notin$ $\left.H_{m}(M)\right\}$. Thus $\operatorname{Soc}(M)=\bigcup_{n<\omega} M_{n}, M_{n} \subseteq M_{n+1}$. By defining $M_{n}$ 's we observe that $M_{n} \cap H_{s_{n}}(M)=0$. This implies that $M$ is $\sigma$-summable.

Theorem 18. If $M$ is a direct sum of $\sigma$-summable QTAGmodules, then so is $L$.

Proof. Let $M=\bigoplus_{i \in I} M_{i}$, where each $M_{i}$ is $\sigma$-summable. Now $L=\bigoplus_{i \in I}\left(L \cap M_{i}\right)$ because $L$ is fully invariant in $M$. Since all $M_{i}$ 's are isotype in $M$, we infer that $L \cap M_{i}$ is large in $M_{i}$, for every $i$. By Theorem 17, $L \cap M_{i}$ are $\sigma$-summable. Thus $L$ is also a direct sum of $\sigma$-summable modules.

Let us recall the following.

Definition 19. A QTAG-module $M$ is $(\omega+1)$-projective if there exists a submodule $N \subseteq \operatorname{Soc}(M)$ such that $M / N$ is a direct sum of uniserial modules.

Remark 20. The submodules of $(\omega+n)$-projective modules are also $(\omega+n)$-projective.

Theorem 21. A QTAG-module $M$ is $(\omega+1)$-projective if and only if its large submodule $L$ is $(\omega+1)$-projective.

Proof. Suppose $L$ is $(\omega+1)$-projective. Therefore there exists a submodule $N \subseteq \operatorname{Soc}(L)$ such that $\operatorname{Soc}(L / N)=\bigcup_{n<\omega}\left(L_{k} / N\right)$, where $L_{k} \subseteq L_{k+1} \subseteq L$ and $L_{k} \cap H_{k}(L) \subseteq N$ for each $k<\omega$. Now $L_{k} \subseteq H^{2}(M)$, for every $k<\omega$. Since

$$
H_{k}\left(H^{2}(L)\right)=H_{t_{k}}\left(H^{2}(M)\right)+\operatorname{Soc}\left(H_{j_{k}}(M)\right),
$$

for some $k \leq j_{k} \leq t_{k}<\omega$, we have

$$
\begin{aligned}
H_{t_{k}}\left(H^{2}(M)\right) & \subseteq H_{k}\left(H^{2}(L)\right) \subseteq H_{j_{k}}\left(H^{2}(M)\right), \\
L_{k} \cap H_{t_{k}}(M) & =L_{k} \cap H_{t_{k}}\left(H^{2}(M)\right) \\
& \subseteq L_{k} \cap H_{k}\left(H^{2}(L)\right)=L_{k} \cap H_{k}(L) \\
& \subseteq N .
\end{aligned}
$$


Therefore the heights of the elements of $L_{k} / N$ are bounded in $M / N$ for all $k<\omega$. Now $(M / N) /(L / N) \cong M / L$ is a direct sum of uniserial modules [6]. Therefore $M / N$ is a direct sum of uniserial modules and $M$ is $(\omega+1)$-projective. The converse is trivial.

The property of being $h$-pure complete is also shared by the large submodules of QTAG-modules.

First we recall the definition of $h$-pure completeness.

Definition 22. A QTAG-module $M$ is $h$-pure complete if, for every subsocle $S \subseteq \operatorname{Soc}(M)$, there is a $h$-pure submodule $N$ of $M$ so that $S=\operatorname{Soc}(N)$. In other words every subsocle supports a $h$-pure submodule of $M$.

Theorem 23. Let $L$ be the large submodule of a QTAG-module $M$. If $M$ is h-pure complete, so is $L$.

Proof. Let $S$ be a subsocle of $L$. Since $S \subseteq \operatorname{Soc}(M), S$ supports a $h$-pure submodule $N$ of $M$. Now $N \cap L$ is also large in $M$ and $N \cap L$ is $h$-pure in $L$. Again $S=\operatorname{Soc}(N) \cap \operatorname{Soc}(L)=\operatorname{Soc}(N \cap L)$, and therefore $L$ is $h$-pure complete.

Corollary 24. A QTAG-module $M$ is h-pure complete if and only if $H_{k}(M)$ is h-pure complete for some fixed but arbitrary positive integer $k$.

Proof. Since $H_{k}(M)$ is large in $M$, it is $h$-pure complete if $M$ is $h$-pure complete. Conversely suppose $H_{k}(M)$ is $h$-pure complete. We shall use transfinite induction to prove the result.

Let $S$ be a subsocle of $M$ such that $S \cap H_{1}(M) \subseteq$ $\operatorname{Soc}\left(H_{1}(M)\right)$ and $S \cap H_{1}(M)=\operatorname{Soc}(N)$ for some $h$-pure submodule $N$ of $H_{1}(M)$. By [7] we can say that there is a $h$ pure submodule $K$ of $M$ such that $H_{1}(K)=N$ and $\operatorname{Soc}(K)=$ $\operatorname{Soc}(N)=\operatorname{Soc}\left(H_{1}(K)\right)$. Now $S \cap H_{1}(M)=\operatorname{Soc}\left(H_{1}(K)\right)$.

We have to show that there exists a $h$-pure submodule $T \subseteq$ $M$ such that $S=\operatorname{Soc}(T)$. We define the submodule $T=K+$ $\left(S \cap \mathrm{H}_{2}(M)\right)$. Now

$$
\begin{aligned}
\operatorname{Soc}(T) & =\operatorname{Soc}\left(K+S \cap H_{2}(M)\right) \\
& =\operatorname{Soc}(K)+\left(S \cap H_{2}(M)\right)
\end{aligned}
$$

because $S \cap H_{2}(M)=\operatorname{Soc}\left(S \cap H_{2}(M)\right)$. Again $\operatorname{Soc}(T)=$ $\operatorname{Soc}(N)+\left(S \cap H_{2}(M)\right) \subseteq S$.

Now

$$
\begin{aligned}
S & =\left(S \cap H_{1}(M)\right) \cup\left(S \cap H_{2}(M)\right) \\
& =\operatorname{Soc}(K) \operatorname{Soc}\left(S \cap H_{2}(M)\right) \\
& =\operatorname{Soc}\left[K \cup\left(S \cap H_{2}(M)\right)\right] \\
& \subseteq \operatorname{Soc}\left[K+\left(S \cap H_{2}(M)\right)\right]=\operatorname{Soc}(T) .
\end{aligned}
$$

Therefore $S=\operatorname{Soc}(T)$. Now,

$$
\begin{aligned}
\operatorname{Soc}(T) \cap H_{t}(M)= & S \cap H_{t}(M) \\
= & {\left[\operatorname{Soc}(K)+\left(S \cap H_{2}(M)\right)\right] } \\
& \cap H_{t}(M) \\
= & {\left[\operatorname{Soc}\left(H_{1}(K)\right)+\left(S \cap H_{2}(M)\right)\right] } \\
& \cap H_{t}(M)=\operatorname{Soc}(K) \cap H_{t}(M) \\
= & \operatorname{Soc}\left(H_{t}(K)\right)=\operatorname{Soc}\left(H_{t}(T)\right) .
\end{aligned}
$$

This implies that $T$ is $h$-pure in $M$.

In the end we state the following unsolved problems.

Problem 25. Is it true that $M$ is a $H F$-module if and only if its large submodule $L$ is?

Problem 26. Is it true that $M$ is a direct sum of closed modules if and only if its large submodule $L$ is?

\section{Competing Interests}

The authors declare that they have no competing interests.

\section{References}

[1] A. Mehdi, M. Y. Abbasi, and F. Mehdi, "On $(\omega+\mathrm{n})$-projective modules," Ganita Sandesh, vol. 20, no. 1, pp. 27-32, 2006.

[2] S. Singh, "Some decomposition theorems in abelian groups and their generalizations," in Ring Theory: Proceedings of the Ohio University Conference, vol. 25, pp. 183-189, Marcel Dekker, New York, NY, USA, 1976.

[3] L. Fuchs, Infinite Abelian Groups. Vol. I, Pure and Applied Mathematics, Vol. 36, Academic Press, New York, NY, USA, 1970.

[4] A. Mehdi, S. A. R. K. Naji, and A. Hasan, "Small homomorphisms and large submodules of QTAG- modules," Scientia Series A: Mathematical Sciences, vol. 23, pp. 19-24, 2012.

[5] M. Z. Khan, "On basic submodules," Tamkang Journal of Mathematics, vol. 10, no. 1, pp. 24-29, 1979.

[6] A. H. Ansari, M. Ahmad, and M. Z. Khan, "Some decomposition theorems on S2-module III," Tamkang Journal of Mathematics, vol. 12, no. 2, pp. 147-154, 1981.

[7] M. Z. Khan, "Modules behaving like torsion abelian groups. II," Mathematica Japonica, vol. 23, no. 5, pp. 509-516, 1979.

[8] S. Singh, "Some decomposition theorems on abelian groups and their generalisations. II," Osaka Journal of Mathematics, vol. 16, no. 1, pp. 45-55, 1979.

[9] A. Mehdi and F. Mehdi, "N-high submodules and h-topology", The South East Asian Journal of Mathematics and Mathematical Sciences, vol. 1, no. 1, pp. 83-88, 2002. 


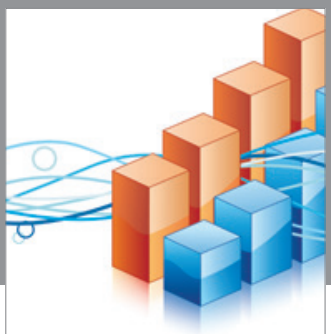

Advances in

Operations Research

vatem alat4

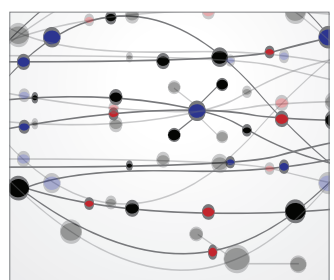

\section{The Scientific} World Journal
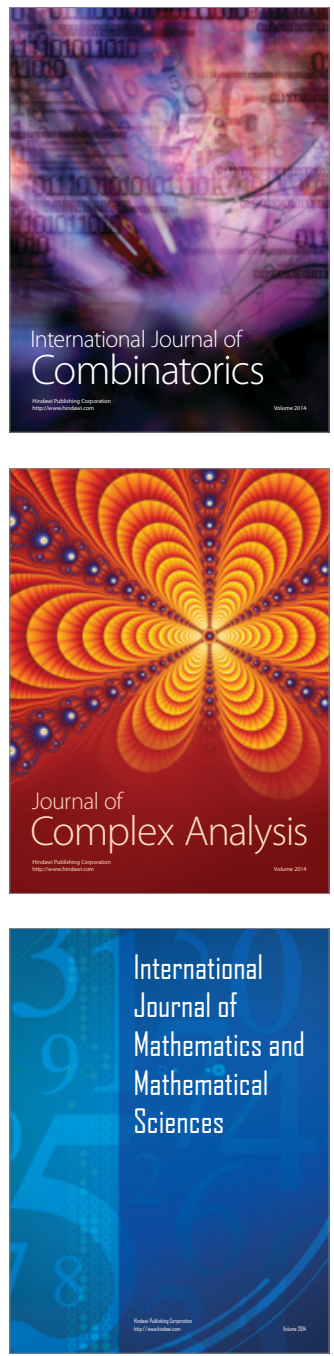
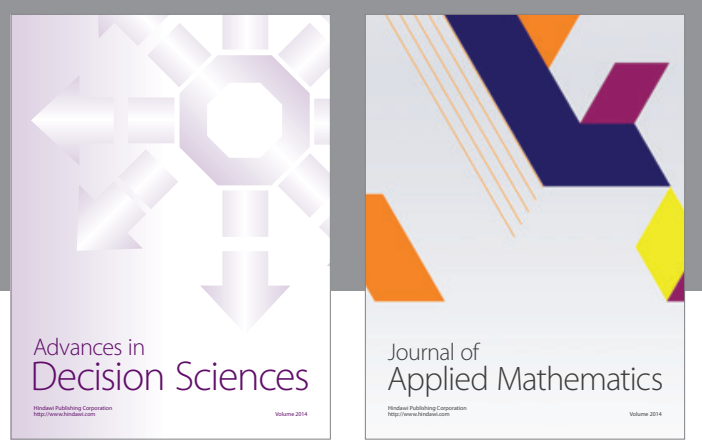

Algebra

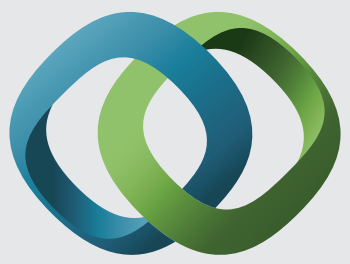

\section{Hindawi}

Submit your manuscripts at

https://www.hindawi.com
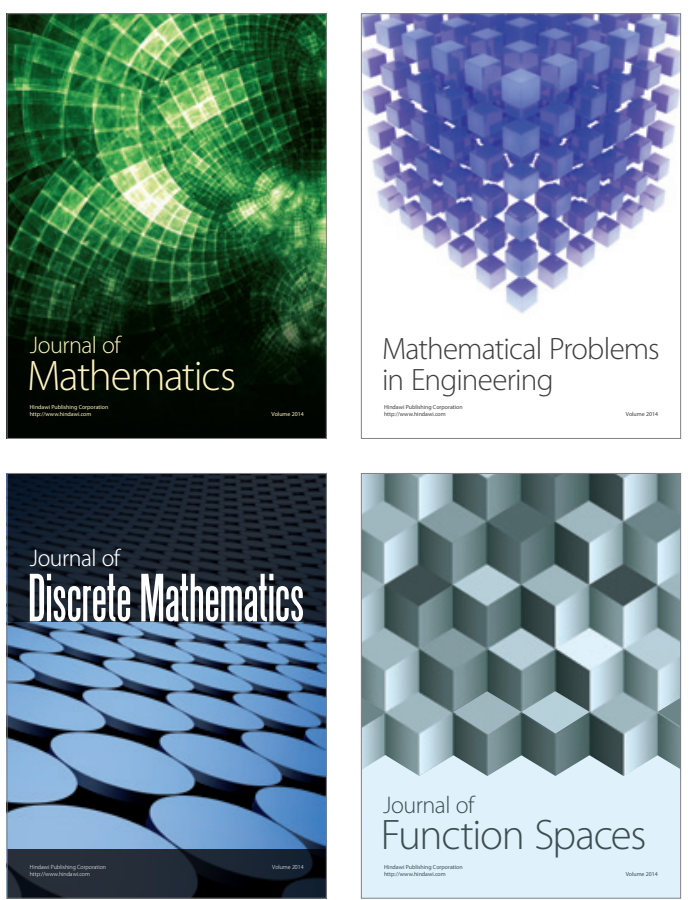

Mathematical Problems in Engineering
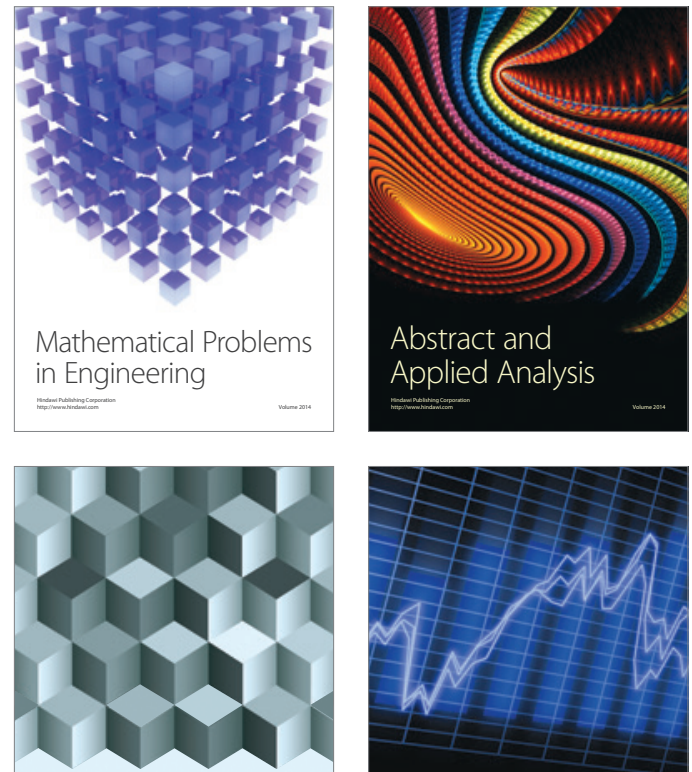

Journal of

Function Spaces

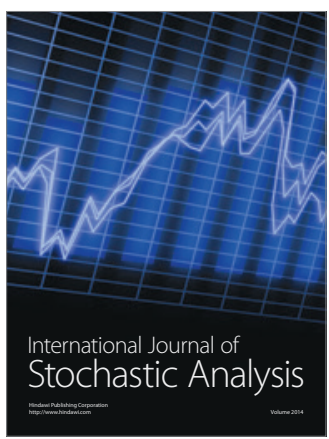

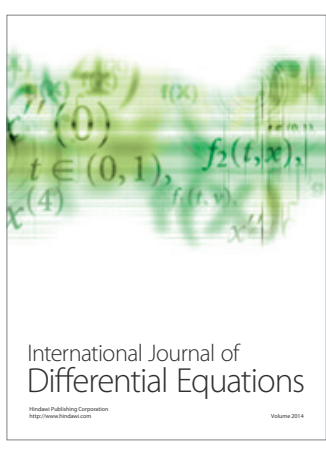
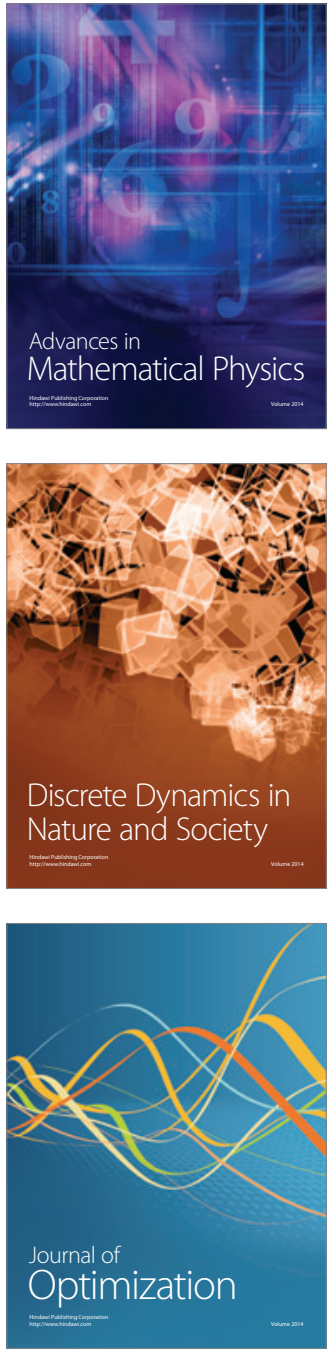